\title{
Folic acid and neural tube defects
}

\author{
RA Minns \\ Royal Hospital for Sick Children, Sciennes Road, Edinburgh EH9 ILF, UK
}

Keywords: neural tube defects; spina bifida; myelomeningocele; paraplegia; folic acid

\section{Embryogenesis}

The primitive nervous system begins as a flat neural plate 2 weeks after conception, having arisen from ectoderm. This plate becomes indented by a longitudinal groove at 20 days with neural folds on the flanks. These folds begin to fuse in the midline forming a cylinder in the middle of the plate and then in a zipper fashion this dorsal closure is promulgated rostrally and caudally resulting in a tubular structure with an open anterior and posterior aperture corresponding to the anterior and the posterior neuropore. At 26 days the anterior neuropore closes and at 29 days the posterior. Factors necessary for the neural tube formation are intrinsic in the neural ectoderm and adjacent mesoderm.

A teratological insult in embryogenesis timed to interfere with the closure of the anterior neuropore will result in anencephalus with failed development of the telencephalic vesicle and the normal cerebral hemispheres are replaced by a mass of undifferentiated cells and abnormal vessels. Anencephalus is responsible for about half of all the neural tube defects and is incompatible with life, and the natural history is for spontaneous abortion, still birth or death shortly after delivery.

Failure of closure of the posterior neuropore results in an exposed spinal cord. When this is subjected to intrinsic hydrodynamic forces and extrinsic mechanical and chemical forces of the intrauterine environment, then progressive destruction of the spinal cord occurs, which results in the full panoply of permanent neurological damage and deformity recognised at birth as spina bifida (myelomeningoceoele). ${ }^{1}$ Although it is predominantly a neuro-ectodermal abnormality there is often involvement of adjacent mesodermal structures. The resultant spina bifida deficit includes life-long handicap from motor and sensory paraplegia, deformity, neuropathic bladder and bowel, Chiari II malformation and hydrocephalus etc, with the extent of the deficit depending predominantly on the pattern and level of spinal cord involvement.

Encephalocoeles are traditionally considered as closed neural tube defects but they are probably a

Correspondence: Dr RA Minns, PhD, FRCP (Ed) post neurulation defect and their precise pathogenesis has yet to be clarified. ${ }^{2}$ They result in failure of fusion of the midline cranial structures with herniation of brain tissue and cerebro-spinal fluid. They are a distinct group of neural tube abnormalities with many more females than males represented, differences in the anterior versus posterior site depending upon race, ie anterior encephalocoeles frequent among Bantus and Thais, and they are associated with a number of characteristic genetic conditions such as the autosomal recessive Meckel-Gruber syndrome, autosomal recessive occipital encephalocoele with myopia and retinal dysplasia, ${ }^{3}$ Joubert syndrome, Walker syndrome etc. They have been reported in association with forty miscellaneous conditions. They may even be deformations rather than malformations arising by extracranial evagination of brain tissue at 20 weeks following raised ventricular pressure in foetal hydrocephalus. ${ }^{4}$

Encephalocoeles result in hydrocephalus, mental retardation with or without microcephaly, epilepsy and ataxia from associated dysplasia in the occipital lobe, cerebellum and cerebral cortex.

In broad terms therefore, neural tube defects result from incomplete neurulation and refer to a wide spectrum of congenital malformations in which separation of the midline vertebral and cranial elements are the common feature, but are usually taken to mean anencephalus, spina bifida and encephalocoeles.

\section{Aetiology of neural tube defects}

It has long been accepted that there was a geneticenvironmental interaction in the causation of neural tube defects. In any large series a positive family history is obtained in about $10 \%$ and we have seen family trees with neural tube defects extending through five generations. If a man or woman with an affected offspring, or either they themselves suffer from the condition, their risk of having a child with neural tube defect is increased tenfold (about 5\% risk). After two such affected pregnancies it is increased 20 times (10$15 \%$ ). The risk can therefore be calculated knowing a detailed family history. These genetic and other epidemiological studies have given rise to the concept 
of high risk 'patients': (1) those with a past history of neural tube defect; (2) maternal age of $<20$ or $>35$ years; (3) parity (primip or grand multip); (4) social class (poverty).

There are 13 genetic loci in rodent models at which mutations are known and three mouse chromosomal models that produce neural tube defects. ${ }^{5}$ In man various chromosome abnormalities, Trisomy 13 and 18 and triploidy and other unbalanced chromosome rearrangements and ring chromosomes, are known to be causes of neural tube defects. Single gene autosomal recessive conditions such as Walker-Warburg, JarcoLevin, Meckel-Gruber, and Robert syndromes are associated with neural tube defects. Autosomal dominant conditions have an association also, such as median facial cleft and the syndrome of anterior sacral meningocoele and anal stenosis.

As well as the genetic risk it has long been known that teratogens are capable of inducing neural tube defects in humans and experimental animals. Copp et $a l^{5}$ discovered from the medical literature more than 60 teratogens that produce neural tube defects in animals, including radiation, maternal hyperthermia, diabetes mellitus, hyper- or hypovitaminosis A, infections such as congenital rubella, toxoplasmosis, cytomegalovirus, and drugs such as aminopterin, valproic acid, aliphatic nitriles, trypan blue, cyclophosphamide and cyanide. A number of environmental hazards have been hypothesised, particularly dietary agents such as soft water, blighted potatoes, nitrate cured corned beef, canned peas treated with magnesium salts, effluent from factories and zinc deficiency.

\section{Periconceptional folate studies}

Hibbard in $1964^{6}$ first suspected that folic acid (a vitamin of the $B$ group) might be involved in the aetiology of neural tube defects, and following this two intervention studies were published which involved vitamin supplementation around the time of conception for women who had already had a child with a neural tube defect. ${ }^{7,8}$ The first study was not randomised and women were given a mixture of eight vitamins including $0.36 \mathrm{mg}$ per day of folic acid. The risk of recurrence in this supplemented group was about one-seventh that of the unsupplemented group. In the second study, which was a small randomised trial of $4 \mathrm{mg}$ per day of folic acid, a significant lower recurrence rate was noted. In a further six observational studies and a non-randomised supplementation study, all with the exception of one showed an association but these studies all either suffered from bias, or were nonrandomised, or were inconclusive. ${ }^{9-15}$

To avoid bias, a large MRC study was proposed and launched in July 1983 with the intention of obtaining at least 2000 pregnancies. ${ }^{16}$ This was an international multicentre double-blind randomised trial involving 33 centres, half in the UK. Women with a previous neural tube defect, not associated with one of the autosomal recessive conditions, who were planning another pregnancy and not already taking supplements, and did not suffer from epilepsy, were included. The effect of supplementation was investigated by a factorial study design which ensured that three-quarters of those taking part received folate or multi-vitamins, or both, with women randomly allocated to one of four supplementation groups: (1) folic acid alone; (2) other vitamins alone (vitamin A, thiamin, riboflavin, B6, vitamin $\mathrm{C}$, vitamin D); (3) both; (4) neither. By April 1991 sufficiently conclusive results were obtained using sequential analysis to warrant stopping the trial. There had been 1195 completed pregnancies with a known outcome. Twenty-seven had a known neural tube defect, six in the folic acid groups and 21 in the other two groups, meaning a $72 \%$ protective effect. It was also concluded that the other vitamins showed no protective effect for neural tube defect, nor did supplementation cause any harm. This careful study assessed compliance and took precautions, by measuring serum folic acid, that women did not additionally supplement themselves. It must be recognised that supplementation with $4 \mathrm{mg}$ per day of folic acid did not prevent all the neural tube defects and these cases did not have unusually low serum folic acid concentrations, and this probably reflects the heterogeneous nature of the condition.

The second significant study from Hungary was a randomised controlled trial, that showed that the occurrence of neural tube defects could be appreciably reduced by giving periconceptional multivitamin supplements which included $0.8 \mathrm{mg}$ of folic acid. ${ }^{17}$ This study was extended to look at the effect on other congenital abnormalities as well, again with a randomised control trial of supplementation with multivitamins (including $0.8 \mathrm{mg}$ of folic acid), or trace elements supplements daily for at least 1 month before conception and at least 2 months after conception. ${ }^{18}$ It was found that periconceptional multivitamin supplements could reduce not only the rate of neural tube defects but the rate of other major genetic syndromic congenital abnormalities such as conotruncal cardiovascular anomalies, defects of the urinary tract, congenital hypertrophic pyloric stenosis, and congenital limb deficiences.

\section{Mechanism of action of folic acid}

The mechanism is still being investigated but several positive facts are emerging. Firstly, it seems unlikely that there is a primary folate deficiency. Kirk et al have shown that mothers carrying foetuses with neural tube defects have a lower B12/folate during pregnancy than mothers carrying normal foetuses, and both folate and B12 are independently predictive of neural tube defects with B12/folate levels well above the deficiency range, suggesting a metabolic block rather than a simple deficiency state. ${ }^{19}$ There is other evidence that $\mathrm{B} 12$ is also involved and others have found an elevated homocysteine level significantly higher for mothers of neural tube defect patients during pregnancy than B12 
matched controls, especially in the lower half of the B12 distribution, ie these cases metabolise homocysteine less well than controls at comparable levels of B12/folate. ${ }^{20,21}$ Adams et al also reported elevated methylmalonic acid values in mothers whose children had neural tube defects, a further marker of B12 insufficiency. ${ }^{22}$

Of the 16 folate dependent enzyme systems, methionine synthase is the only one which requires $\mathrm{B} 12$ as well as folate, and a defect in methionine synthase would lead to this increased level of homocysteine suggesting an abnormality of methionine synthase together with its co-factor B 12 could be the responsible metabolic defect. ${ }^{20}$

Other situations that can result in an increased homocysteine level might also be implicated and a mutation has been identified $(677 \mathrm{C} \rightarrow \mathrm{T}$ mutation) in the 5,10-methylenetetrahydrofolate reductase (MTHFR) gene on chromosome 1p 36.3, which is present in $13 \%$ of neural tube defect patients, $16 \%$ of mothers, and $10 \%$ of fathers, compared to $5 \%$ of controls who are homozygous for this. ${ }^{23}$ This mutation results in a decreased MTHFR activity, a low plasma folate (another marker of abnormal methionine synthase activity) and high levels of homocysteine. This mutation can be regarded as a genetic risk factor for spina bifida and supports the hypothesis of defective folate metabolism in at least a subset of mothers with neural tube defects. ${ }^{24}$

Cystathionine synthesase abnormalities can also raise homocysteine and this has also been reported in a minority of patients with neural tube defects. ${ }^{25}$ Overcoming an abnormality in homocysteine metabolism, particularly an abnormality of methionine synthase by folic acid supplementation, is presently the favoured postulated mechanism of action of folic acid. Methionine synthase is pivotal in methylation reactions for producing myelin basic protein and in making tetrahydrofolate for DNA synthesis, either of which may be the responsible pathway for producing neural tube defects. ${ }^{26}$

In experimental animals various results have been found, for example in mice folate did not prevent neural tube defects but methionine did. ${ }^{27}$ The folic acid antagonists aminopterin and methotrexate did not cause neural tube defects but rather limb deficiencies. Valproic acid results in $1-2 \%$ incidence of spina bifida possibly by interacting with embryonic folate metabolism. ${ }^{28}$ The 'curly tail mouse' has been studied extensively to determine agents which prevent neural defects in embryos and prevention has been found with retinoic acid, inositol, hydroxyurea, mitomycin C, 5-flurouracil, cytosine arabinoside, possibly riboflavin, vitamin $\mathrm{C}$, vitamin $\mathrm{D} 2$. But no prevention was seen with folic acid, folinic acid, pregnavite forte $\mathrm{F}, \mathrm{B} 6, \mathrm{~B} 12$, triamcinolone, cyclohexamide, zinc, homocysteine, methionine and thymidine. $^{29}$ Ovulatory drugs such as chlomiphene and hormones do not cause an increased risk of neural tube defect. ${ }^{30}$

\section{Optimal delivery method of folic acid}

Conclusive evidence from the MRC and Hungarian studies means that prevention is effective for women who have not had a foetus with a neural tube defect as well as for those who have, ${ }^{31}$ and a clear policy is necessary to prevent primary occurrences and recurrences. This could be accomplished by either supplementation or food fortification, and because of the public health implications of the latter option the advantages and disadvantages of each are discussed.

Supplementation has been recommended by the Expert Advisory Group to prevent recurrences of neural tube defects by taking a daily dose of $4 \mathrm{mg}$ before conception and continued until the 12th week of pregnancy. ${ }^{32}$ This advice was also for the offspring of women or men with spina bifida. Only $5 \mathrm{mg}$ tablets are available and these only on prescription. To prevent the first occurrence of a neural tube defect extra folate was recommended for all women prior to conception and during the first 12 weeks of pregnancy, and the suggested ways of achieving this were by extra intake of folate rich foods such as green leafy vegetables, not over cooking, and by eating foods fortified with folic acid, and additionally taking a food supplement. The recommended supplemental dose to prevent occurrences was $0.4 \mathrm{mg}$ of folic acid daily when they begin trying to conceive or on the suspicion of pregnancy, or if the women recognises the possibility of pregnancy following unprotected sexual intercourse until the 12th week of pregnancy. In any case at the suspicion of pregnancy supplementation should commence at once. $0.4 \mathrm{mg}$ tablets are available without prescription from chemists or health food stores.

The advantages of supplementation are that it targets only the population who are at risk and as such the type of medication, its dose and the target group can be clearly defined.

The disadvantages of supplementation are:

(1) That a large percentage of pregnancies are unplanned and this includes about $95 \%$ of the pregnancies that end in a neural tube defect.

(2) The practical difficulties are considerable in attempting to give all women supplementation before falling pregnant, particularly those in a low socio-economic group who are at a higher risk.

(3) There must be a public health strategy in conjuction with the supplementation about the necessity of it, dietary advice etc.

(4) Although small, there is still a financial price to pay for the supplement.

(5) The theoretical and unknown risks of providing supplementation to populations who already have a low incidence of neural tube defects.

(6) The background dietary folic acid being consumed before supplementation will be variable and thus the total daily intake variable.

(7) In 1995, 3 years after the Department of Health 
recommendations and a public awareness campaign, there was still widespread ignorance about the need to supplement by most people. ${ }^{33,34}$

It is likely therefore that supplementation would not be sufficiently effective and is beset by a number of practical difficulties. Food fortification was therefore considered.

It is assumed that in the UK the dietary folate intake is now on average $0.2 \mathrm{mg}$ per day. ${ }^{35}$ To bring dietary folate to the necessary $0.6 \mathrm{mg}$ per day by a change of diet would be difficult. Some breakfast cereals and at least one brand of bread are already fortified with folic acid to a reasonable level (one slice of bread contains $0.05 \mathrm{mg}$ of folic acid). This dose is unlikely to cause any problems and the Expert Advisory Group recommended that fortification was restricted to cereals and bread, but that the range of fortified cereals and bread be increased so there was a greater variety. They did not, however, recommend universal fortification thus allowing the important choice of unfortified foods to remain available.

The advantages of fortification are:

(1) It ensures compliance in all of the population at risk by consumption by the whole population.

(2) Collective action has always been an essential part of public health achievements.

(3) There are minimal side effects, even in large doses it is not harmful and folic acid is water soluble which means it is not stored and what is not needed is excreted and does not affect the foetus, unlike other vitamins such as vitamin $\mathrm{A}$.

(4) Primary folate deficiency exists in the population and would be treated by this fortification policy.

(5) Unfortified bread would still be available although it would have to be labelled as the exception.

The disadvantages of fortification are:

(1) The theoretical concern in older patients with undiagnosed pernicious anaemia given folic acid which may alleviate the anaemia while failing to detect signs of subacute combined degeneration of the cord, although this may then present sooner and timely treatment with B12 may even reverse the neuropathy. ${ }^{36}$.

(2) It may result in EEG abnormalities in epileptic patients who may require more anti-epileptic drugs.

(3) Patients taking folic acid antagonists are potentially at risk.

(4) It might be considered that fortification infringes the individual's rights, that is, the power of the state overriding the rights of the individual and following on from that, the concept of coersive healthism for those who do not readily follow the government line.

(5) A theoretical risk of overdosing if additional complementation with folic acid was added.

(6) Lifestyle and cultural differences would necessitate guidance for all ethnic groups about foods to be consumed to achieve adequate levels. For example, Asian communities do not eat bread and would require fortification of chapati flour with folic acid.

(7) Fortification with folate alone may be insufficient and there remains the possibility that other multivitamins might be responsible for preventing malformations together with the possibility that if B12 was added as a fortified constituent it would necessitate lower dose of fortification with folate.

A new public awareness campaign was launched on 26th February 1996 to run for the next 2-3 years. The question of whether widespread fortification should be arranged with food manufacturers on a voluntary basis or whether it should be compulsory is still to be decided, but there are a growing number of advocates for strengthening the voluntary recommendations to a compulsory policy at an early stage, eg at milling sites rather than at manufacture. ${ }^{37}$

In the United States a new fortification order requires folic acid be added to all enriched foods from 1st January 1998. The Public Health Service recommended in 1992 that all women of childbearing age should eat $0.4 \mathrm{mg}$ of folic acid daily and by fortifying grain products they were making it easier to achieve adequate folate levels in the diet. It will be added to most enriched breads, flours, cornmeals, pastas, rice and other grain products. These represent the staple diet of most of the US population. The fortification policy is designed to keep daily intakes of folic acid below $1 \mathrm{mg}$.

\section{Declining prevalence of neural tube defects}

The birth prevalence of neural tube defect appears to have been declining in many parts of the world since $1970 .^{38}$

In Scotland the decline has paralleled the institution of antenatal screening. The 'adjusted birth prevalence', ie birth plus terminations for neural tube defects, fell by more than a third between 1974 and 1982. The actual birth prevalence, ie excluding terminations, fell by $63 \%$ for spina bifida and $91 \%$ for anencephaly suggesting that antenatal screening was responsible for most of the decline in anencephalic prevalence, and the decline in spina bifida prevalence was due to other factors. Antenatal screening probably accelerated a trend or natural fall. ${ }^{39}$

In South Australia between 1966 and 1991, the total prevalence remained stable but prenatal diagnosis and termination resulted in an $84 \%$ fall in birth prevalence during these years, with screening detecting $85 \%$ of cases. $^{40}$

There does appear to have been a natural fall in prevalence in the Republic of Ireland where there was no screening or termination as well as in other countries before screening programmes commenced and this natural fall in prevalence has been exacerbated by screening programmes. 


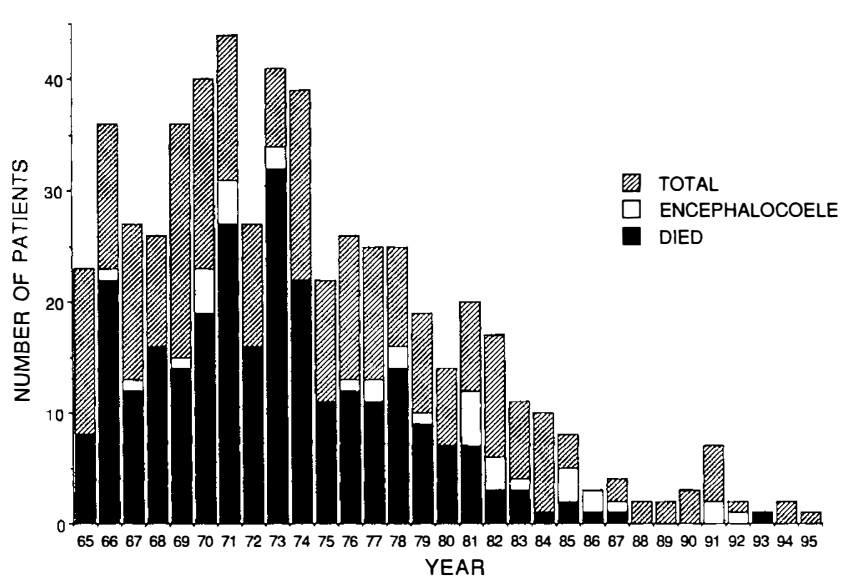

Figure 1 The figure illustrates birth prevalence of cases of spina bifida (myelomeningocoele and meningocoele) and encephalocoeles admitted to a Regional Paediatric Neurology Service at RHSC Edinburgh over a 31 year period, additionally showing the number dying in infancy. The impact of AFP/ultrasound screening and termination from 1975 is dramatic but the consistently small numbers since 1986 conceals the total prevalence. From 1986-1994 inclusive, the total live births using ICD-9 statistics for anencephaly, spina bifida and encephalocoeles was 22 . Over the same time period, terminations and stillbirths for neural tube defects totalled 86 , showing that screening/termination was responsible for most of the decline, however the yearly numbers of terminations and stillbirths have also declined steadily over the same time period from 18 per year in 1986 to four per year in 1994

Secondary prevention through screening with the option of termination has been available for the last 20 years. All cases of anencephaly and $66 \%$ of cases of spina bifida will be identified by alphafetoprotein (blood and aminiotic) and ultrasound scan. Termination together with a selective policy postnatally has been responsible for the main decline in prevalence. Primary preventative measures therefore in preventing the development of neural tube defect in the embryo by periconceptional vitamins would have an additional and overlapping contribution to the decline.

The birth prevalence of myelomeningocoele and encephalocoeles admitted to a regional paediatric neurology service in Edinburgh over a 30 year period from 1965-1994 showed the dramatic impact of antenatal screening from 1975 with a steady decline to about 1985 . The virtually static nature of our birth prevalence since then is largely due to the continued effectiveness of screening and termination, and it must be remembered that there will always be a limit to the effectiveness of antenatal screening/termination because not all pregnant women avail themselves of all antenatal services. The impact of periconceptional vitamins supplementation is too early to say, but there does appear to be a decline in the total prevalence, ie live births plus terminations and stillbirths using ICD-9 statistics. For the Lothian Region in 1985 the total prevalence of neural tube defects was 22, and since 1993 has been less than 10. In the UK approximately 2000 would still occur although three-quarters end in stillbirth or termination and most of these would be prevented by fortification.

Prior to the periconceptional vitamin study the alternatives were for screening and termination (depending on parental wishes) of all AFP positive foetuses or those detected by ultrasound. There is no possibility of predicting in utero the degree of neurological deficit and Hunt with a series of 117 spina bifida adolescents between 16 and 20 years of age, who were likely to have had an elevated AFP level, showed that some $7 \%$ of these had little or no disability. ${ }^{41}$ The AFP and ultrasound are not discriminating and if positive all are offered termination. The 'verebral level' and the 'lesion level' and the degree of hydrocephalus from the ultrasound are not good indicators of subsequent handicap and vice versa. Low level lesions may have a very significant handicap. What the ultrasound cannot do is detect the motor or sensory neurological level, although attempts have been made to deduce levels from flexion and extension of the knee. Since it is not possible to detect the severity of neurological damage in-utero a termination policy is an imprecise secondary prevention of neural tube defects so that all lesions functionally good or bad with an elevated AFP are offered termination.

Postnatally many units have used a selective policy based on modified Lorber criteria for operation or not. ${ }^{42}$ It must be remembered that the operation is not curative and, our own unit has a selective postnatal policy but with the caveat that a U-turn in management policy is practised if the child survives.

Secondary prevention (termination of affected foetuses regardless of severity, together with postnatal 'selecting out') is ethically less acceptable than primary prevention of the neural tube defect, together with the possibility that in the future, in-utero repair of the open spina bifida may be feasible for those neural tube defects not prevented by periconceptional vitamins. ${ }^{1}$

A number of unanswered questions remain. For example the dosage, at present a $5 \mathrm{mg}$ tablet, is made by a number of manufacturers, it is cheap and there is unlikely to be pressure to develop a $4 \mathrm{mg}$ tablet for daily consumption, but could it be that the original $0.36 \mathrm{mg}$ is enough or $0.8 \mathrm{mg}$ as suggested in the Hungarian study. Neither is it known how long before conception this is required and will dietary and supplementary folic acids be compatible, and will the benefits of folic acid be the same in populations with a previously low prevalence.

Periconceptional folic acid supplementation and fortification represents a major advance in the prevention of neural tube defects in a primary preventative way. The MRC trial which brought this about is a milestone which, with or without termination, will make a very dramatic influence on the number of infants likely to be born with this major congenital malformation. 


\section{Acknowledgements}

I am grateful to Dr E Sowler, Principal Medical Officer, Department of Health, The Scottish Office and Dr Marion Bain, Consultant in Public Health Medicine, Information and Statistics Division, Trinity Park House, Edinburgh.

\section{References}

1 Meuli M, Meuli-Simmen C, Hutchins GM et al. In utero surgery rescues neurological function at birth in sheep with spina bifida. Nature Med 1995; 1: $342-347$.

2 Campbell LR, Dayton DH, Sohal GS. Neural tube defects: A review of human and animal studies on the etiology of neural tube defects. Teratology 1986; 34: 171-187.

3 Knoblock WH, Layer JM. Retinal detachment and encephalocele. J Pediatr Ophthalmol 1971; 8: 181.

4 Evrard P, Lyon G, Gadisseux JF. Le developpement prenatal du systeme nerveux et ses perturbations: mecanismes generaus Progres en neonatologie vol. 4 Basel, NY: Karger, 1984; pp $63-$ 69.

5 Copp AJ, Brook FA, Estibeiro JP, Shum ASW. The embryonic development of mammalian neural tube defects. Prog Neurobiol 1990; 35: 363 - 403 .

6 Hibbard BM. The role of folic acid in pregnancy. $J$ Obstet Gynaecol Brit Commwlth 1964; 71: 529-542.

7 Smithells RW, Shephard S, Schorah CJ et al. Possible prevention of neural-tube defects by periconceptional vitamin supplementation. Lancet 1980; i: 339-340.

8 Laurence KM, James $\mathrm{N}$, Miller $\mathrm{MH}$ et al. Double-blind randomised controlled trial of folate treatment before conception to prevent recurrence of neural-tube defects. Br Med J 1981; 282: $1509-1511$.

9 Laurence KM, James N, Miller MH et al. Increased risk of recurrence of pregnancies complicated by fetal neural tube defects in mothers receiving poor diets, and possible benefit of dietary counselling. Br Med J 1980; 281 1592-1594.

10 Winship KA, Cahal DA, Weber JCP et al. Maternal drug histories and central nervous system anomalies. Arch Dis Child 1984; 59: $1052-1060$

11 Mulinare J, Cordero JF, Erickson D et al. Periconceptional use of multivitamins and the occurrence of neural tube defects. JAMA 1988; 260: 3141 - 3145 .

12 Mills JL, Rhoads GG, Simpson JL et al. The national Institute of Child Health and Human Development Neural Tube Defect Study Group. The absence of a relation between the periconceptional use of vitamins and neural-tube defects. $N$ Engl J Med 1989; 321: 430 - 435 .

13 Bower C, Stanley FJ. Dietary folate as a risk factor for neuraltube defects: evidence from a case-control study in Western Australia. Med J Aust 1989; 150: 613-618.

14 Milunsky A, Jick H, Jick SS et al. Multivitamin/folic acid supplementation in early pregnancy reduces the prevalence of neural tube defects. JAMA 1989; 262: $2847-2852$.

15 Vergel RG, Sanchez LR, Herdero BL et al. Primary prevention of neural tube defects with folic acid supplementation: Cuban experience. Prenat Diagn 1990; 10: 149- 152.

16 MRC Vitamin Study Research Group. Prevention of neural tube defects: results of the Medical Research Council Vitamin Study. Lancet 1991; 338: $131-137$.

17 Czeizel AE, Dudas I. Prevention of the first occurrence of neural tube defects by periconceptional vitamin supplementation. $N$ Engl J Med 1992; 327: 1832 - 1835 .

18 Czeizel AE. Prevention of congenital abnormalities by periconceptional multivitamin supplementation. $B r$ Med J 1993; 306: $1645-1648$
19 Kirke PN, Molloy AM, Daly LE et al. Maternal plasma folate and vitamin $B_{12}$ are independent risk factors for neural tube defects. $Q J$ Med 1993; 86: $703-708$.

20 Mills JL, McPartlin JM, Kirke PN et al. Homocysteine metabolism in pregnancies complicated by neural-tube defects. Lancet 1995; 345: 149-151.

21 Steegers-Theunissen RPM, Boers GHJ, Trijbels FJM et al Maternal hyperhomo-cysteinemia: a risk-factor for neural-tube defects? Metabolism 1994; 43: 1475 - 1480.

22 Adams MJ, Khoury MJ, Stevenson R et al. Midtrimester serum methylmalonic acid as a risk marker for neural tube defects. Society for Epidemiologic Research, 1993 Annual Meeting, Keystone, Colorado (abstr).

23 Goyette P, Sumner JS, Milos et al. Human methylenetetrahydrofolate reductase: isolation of cDNA, mapping and mutation identification. Nature Genet 1994; 7: 195-200.

24 van der Put N, Steegers-Theunissen RPM, Frosst P et al. Mutated methylenetetrahydrofolate reductase as a risk factor for spina bifida. Lancet 1995; 346: 1070-1071.

25 Steegers-Theunissen RP, Boers GH, Trijbels FJ et al. Neuraltube defects and derangement of homocysteine metabolism. $N$ Engl J Med 1991; 324: 199-200.

26 Essien FB, Wannberg SL. Methionine but not folinic acid or vitamin B-12 alters the frequency of neural tube defects in Axd mutant mice. $J$ Nutr 1993; 123: $27-34$.

27 Coelho CND, Weber JA, Klein NW et al. Whole rate embryos require methionine for neural tube closure when cultured on cow serum. J Nutr 1989; 119: 1716-1725.

$28 \mathrm{Nau}$ H. Valproic acid-induced neural tube defects. Ciba Found Symp (Netherlands) 1994; 181: 144-152.

29 Seller MJ. Vitamins, folic acid and the cause and prevention of neural tube defects. Ciba Found Symp (Netherlands) 1994; 181: $161-173$

30 Werler MM, Louik C, Shapiro S et al. Ovulation induction and risk of neural tube defects. Lancet 1994; 344: 445-446.

31 Wald NJ. Folic acid and neural tube defects: the current evidence and implications for prevention. In: Neural Tube Defects. Chichester: Wiley, 1994: pp 192-211. (CIBA Foundation Symposium 181).

32 Expert Advisory Group. Folic acid and the prevention of neural tube defects. Department of Health 1992. Published by Health Publications Unit, Heywood, Lancashire.

33 Sutcliffe M, Schorah CJ, Perry A et al. Prevention of neural tube defects Lancet 1993; 342: 1174

34 Clark NAC, Fisk NM. Minimal compliance with the Department of Health recommendation for routine folate prophylaxis to prevent fetal neural tube defects. Br J Obstet Gynaecol 1994; 101: $709-710$.

35 Ministry of Agriculture, Fisheries and Food. Household Food Consumption and Expenditure 1990 London: HMSO, 1991.

36 Wald NJ, Bower C. Folic acid, pernicious anaemia, and prevention of neural tube defects. Lancet 1994; 343: 307.

37 Wald NJ, Bower C. Folic acid and the prevention of neural tube defects. A population strategy is needed. Br Med J 1995; 310: $1019-1020$.

38 Elwood JM, Elwood JH. Epidemiology of Anencephalus and Spina Bifida Oxford University Press: Oxford 1980.

39 Carstairs V, Cole S. Spina bifida and anencephaly in Scotland. $\mathrm{Br}$ Med J 1984; 289: 1182-1184.

40 Chan A, Robertson EF, Haan EA et al. Prevalence of neural tube defects in South Australia, 1966-91: effectiveness and impact of prenatal diagnosis. Br Med J 1993; 307: 703-706.

41 Hunt GM. Open spina bifida; outcome for a complete cohort treated unselectively and followed into adulthood. Dev Med Child Neurol 1990; 32: $108-118$

42 Lorber J. Results of treatment of myelomeningocele. An analysis of 524 unselected cases with special reference to possible selection for treatment. Dev Med Child Neurol 1971; 13: 279-303. 